Pacific Journal of Mathematics

AN EXTENSION OF SOME RESULTS OF TAKESAKI IN THE
REDUCTION THEORY OF YON NEWMAN ALGEBRAS 


\title{
AN EXTENSION OF SOME RESULTS OF TAKESAKI \\ IN THE REDUCTION THEORY OF VON NEUMANN ALGEBRAS
}

\author{
George A. Elliott
}

\begin{abstract}
Briefly, the results in this paper are that both for measurable fields of von Neumann algebras and for families of measurable fields of operators, pointwise isomorphism implies isomorphism.

In the special case when half the measurable fields considered are constant, these results were established by Takesaki. If the Borel space on which the fields are defined is standard, the results can be established by classical means; in the case considered by Takesaki they are due to von Neumann.

For the results of the present paper, two new tools seem to be needed. The first is a measurable choice theorem of Aumann which generalizes the classical one. This has already been applied to reduction theory by Flensted-Jensen. The second is a criterion for a von Neumann algebra containing the diagonal operators to be decomposable: it should consist of decomposable operators. This answers a question of Dixmier.
\end{abstract}

We shall use the terminology of reduction theory developed in [2], Chapitre II.

2. Lemma (Aumann). Let $T$ be a Borel space and let $X$ be a standard Borel space. Let $G$ be a Borel subset of $T \times Y$ such that the projection of $G$ onto $T$ is all of $T$. Let there be given a finite measure on $T$. Then there exists a measurable map $g: T \rightarrow X$ such that $(t, g(t)) \in G$ for almost all $t \in T$.

Proof. See [1]. The proof is by reduction to the case that $T$ is standard.

3. Theorem. Let $T$ be a Borel space, and suppose given a finite measure on $T$ and a measurable field of Hilbert spaces on $T$ with direct integral $H$. Let $A$ and $B$ be decomposable von Neumann algebras in $H$. If for each $t \in T$ there is a spatial isomorphism of $A(t)$ onto $B(t)$ then there exists a decomposable spatial isomorphism of $A$ onto $B$. This statement also holds with the word "spatial" removed. 
Proof. The proof of the first assertion is the same as the proof of Lemma 2 on page 179 of [2], with the exception that 2 above is used instead of the more well known measurable choice theorem for standard measures.

The second assertion is reduced to the first by tensoring with the scalars on a separable infinite dimensional Hilbert space, just as in Theorem 3 of [4].

4. Lemma. Let $T$ be a Borel space, and suppose given a finite measure on $T$ and a measurable field of Hilbert spaces on $T$ with direct integral $H$. Then a von Neumann algebra in $H$ containing the diagonal operators is decomposable if it consists of decomposable operators.

This answers affirmatively the question on page 174 of [2].

Proof. We may suppose that the field of Hilbert spaces is constant. By [2], page 178, Corollaire, it then follows that the algebra of all decomposable operators is spatially isomorphic to $Z \otimes B$ with $Z$ a commutative algebra and $B$ the algebra of all operators on a separable Hilbert space. Since the algebra of diagonal operators is countably decomposable (the measure of $T$ is finite), so is $Z$; therefore both $Z$ and $B$ and hence also $Z \otimes B$ have a countable separating set of vectors.

Let $\xi_{1}, \xi_{2}, \cdots$ be a countable separating set of vectors for the algebra of all decomposable operators, such that $\Sigma\left\|\xi_{i}\right\|^{2}<\infty$. Then for any operator $x$ we have $\left(\Sigma\left\|x \xi_{i}\right\|^{2}\right)^{1 / 2}<\infty$. On the algebra of decomposable operators this expression defines a norm, which on bounded sets determines the strong topology. We shall denote this norm by $N$.

Let $A$ be a von Neumann algebra containing the algebra of diagonal operators, and consisting of decomposable operators. To show that $A$ is decomposable, we must show that $A$ is countably generated over the algebra of diagonal operators ([2], page 174, Théorème 2). Writing as before the algebra of decomposable operators as $Z \otimes B$ with $Z$ commutative and $B$ a type $I_{n}$ factor, $n$ countable, let $x_{1}, x_{2}, \cdots$ be a sequence strongly dense in the unit ball of $1 \otimes B$. For each $k=1,2, \cdots$ let $y_{k}$ be an element of $A$ which is at minimal distance from $x_{k}$ with respect to the norm $N$ of the preceding paragraph (such $y_{k}$ exists because bounded weakly closed sets of $A$ are weakly compact, and $N$ is weakly lower semicontinuous). Then $y_{1}, y_{2}, \cdots$ generate $A$ over the algebra of diagonal operators. For if $e$ is a diagonal projection then for each $k=1,2, \cdots$ the distance from $e y_{k}$ to $e x_{k}$ with respect to $N$ is minimal. Hence, if $e_{1}, \cdots, e_{p}$ are 
diagonal projections with sum 1 , and if $k_{1}, \cdots, k_{p}=1,2, \cdots$ then the distance of $e_{1} y_{k_{1}}+\cdots+e_{p} y_{k_{p}}$ to $e_{1} x_{k_{1}}+\cdots+e_{p} x_{k_{p}}$ with respect to $N$ is minimal. The assertion follows, because the operators $e_{1} x_{k_{1}}+\cdots+e_{p} x_{k_{p}}$ as above are strongly dense in the unit ball of decomposable operators, and the strong topology on this unit ball is metrized by $N$.

5. Theorem. Let $T$ be a Borel space, and suppose given a finite measure on $T$ and a measurable field of Hilbert spaces on $T$ with direct integral $H$. Let $\left(x_{i}\right)$ and $\left(y_{i}\right)$ be families of decomposable operators in $H$. If for each $t \in T$ the families $\left(x_{i}(t)\right)$ and $\left(y_{i}(t)\right)$ are simultaneously unitarily equivalent, then $\left(x_{i}\right)$ and $\left(y_{i}\right)$ are simultaneously unitarily equivalent, with the equivalence implemented by a decomposable unitary operator.

Proof. Suppose first that the families $\left(x_{i}\right)$ and $\left(y_{i}\right)$ are countable. Then the conclusion may be deduced as in A 82, page 348 of [3], using again 2 instead of the classical measurable choice theorem.

If the families $\left(x_{i}\right)$ and $\left(y_{i}\right)$ are not countable, by 4 it is still true that the von Neumann algebra $A$ generated by the $x_{i}$ and the diagonal operators is decomposable. It follows that there exists a countable family $w_{1}, w_{2}, \cdots$ in $A$ generating $A$ over the diagonal operators. We may suppose that the $x_{i}$ form a sub involutive algebra, containing the diagonal operators. Then the $x_{i}$ are strongly dense in $A$, and the $x_{i}$ of norm $\leqq 1$ are strongly dense in the unit ball of $A$. As shown in the proof of 4, the strong topology on the unit ball of $A$ is metrizable. We may suppose that $w_{1}, w_{2}, \cdots$ lie in the unit ball of $A$. Then there exists a countable subfamily of $\left(x_{i}\right)$ which generates $A$ over the diagonal operators (namely, the union of sequences converging strongly to each $\left.w_{k}, k=1,2, \cdots\right)$. By the first paragraph of the proof this countable subfamily of $\left(x_{i}\right)$ is simultaneously unitarily equivalent to the corresponding subfamily of $\left(y_{i}\right)$, by a decomposable unitary operator, say $v$.

We claim that $v x_{i} v^{*}=y_{i}$ for every $i$. The subfamily of $\left(x_{i}\right)$ such that $v x_{i} v^{*}=y_{i}$ contains the diagonal operators and also a set (the above countable subfamily) which generates $A$ over the diagonal operators. It therefore contains a sub involutive algebra dense in $A$. By metrizability of the strong topology on bounded sets, this subfamily is closed under strong limits (use Proposition 4, page 160 of [2]). It follows that $v x_{i} v^{*}=y_{i}$ for all $i$.

6. REMARKs. Once 5 has been reduced by use of 4 to the case that the families are countable, the proof can also be finished by a variant of the method of Takesaki, in [4] (in which not just a measur- 
able choice but a Borel choice is made).

On the other hand, although Takesaki was able to prove his special case of 3 by a Borel choice argument, the author does not see how to extend this approach and was forced to be content in the proof of 3 with making a measurable choice.

\section{REFERENCES}

1. R. J. Aumann, Measurable utility and measurable choice theorem (Research program in Game Theory and Mathematical Economics, No. 30), Hebrew University, Jerusalem, 1967.

2. J. Dixmier, Les algèbres d'opérateurs dans l'espace hilbertien, $2^{e}$ édition, Gauthier-Villars, Paris, 1969.

3. — Les $C^{*}$-algèbres et leurs représentations, $2^{e}$ édition, Gauthier-Villars, Paris, 1969.

4. M. Takesaki, Remarks on the reduction theory of von Neumann algebras, Proc. Amer. Math. Soc., 20 (1969), 434-438.

Received December 22, 1970.

QueEn's University at Kingston, CANADA

AND

The Institute for Advanced Study, Princeton, New Jersey 


\section{PACIFIC JOURNAL OF MATHEMATICS}

\section{EDITORS}

H. SAMELSON

Stanford University

Stanford, California 94305

C. R. HobBY

University of Washington

Seattle, Washington 98105
J. DugundjI

Department of Mathematics

University of Southern California

Los Angeles, California 90007

RICHARD ARENS

University of California

Los Angeles, California 90024

\section{ASSOCIATE EDITORS}

E. F. BECKENBACH

B. H. NeUMaNN

F. WOLF

K. YoshidA

\section{SUPPORTING INSTITUTIONS}

UNIVERSITY OF BRITISH COLUMBIA

CALIFORNIA INSTITUTE OF TECHNOLOGY

UNIVERSITY OF CALIFORNIA

MONTANA STATE UNIVERSITY

UNIVERSITY OF NEVADA

NEW MEXICO STATE UNIVERSITY

OREGON STATE UNIVERSITY

UNIVERSITY OF OREGON

OSAKA UNIVERSITY
UNIVERSITY OF SOUTHERN CALIFORNIA STANFORD UNIVERSITY

UNIVERSITY OF TOKYO

UNIVERSITY OF UTAH

WASHINGTON STATE UNIVERSITY UNIVERSITY OF WASHINGTON

$\stackrel{*}{*} \stackrel{*}{*} \stackrel{*}{*}$ AMERICAN MATHEMATICAL SOCIETY
NAVAL WEAPONS CENTER 


\section{Pacific Journal of Mathematics}

Vol. 39 , No. 1

May, 1971

Charles A. Akemann, A Gelfand representation theory for $C^{*}$-algebras ....

Sorrell Berman, Spectral theory for a first-order symmetric system of

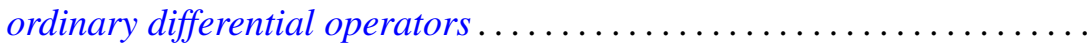

Robert L. Bernhardt, III, On splitting in hereditary torsion theories ........

J. L. Brenner, Geršgorin theorems, regularity theorems, and bounds for determinants of partitioned matrices. II. Some determinantal identities ..........................................

Robert Morgan Brooks, On representing $F^{*}$-algebras .............. 51

Lawrence Gerald Brown, Extensions of topological groups........... 71

Arnold Barry Calica, Reversible homeomorphisms of the real line ........ 79

J. T. Chambers and Shinnosuke Oharu, Semi-groups of local Lipschitzians in

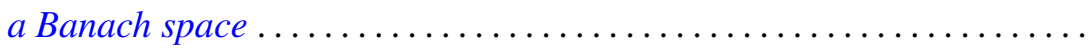

Thomas J. Cheatham, Finite dimensional torsion free rings .............

Byron C. Drachman and David Paul Kraines, A duality between

transpotence elements and Massey products ...................

Richard D. Duncan, Integral representation of excessive functions of a

Markov process ......................................

George A. Elliott, An extension of some results of Takesaki in the reduction

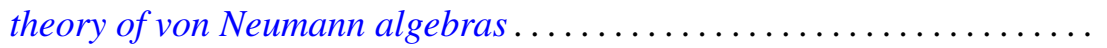

Peter C. Fishburn and Joel Spencer, Directed graphs as unions of partial

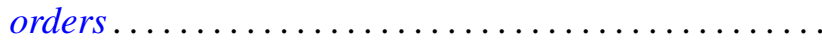

Howard Edwin Gorman, Zero divisors in differential rings ...

Maurice Heins, A note on the Löwner differential equations...

Louis Melvin Herman, Semi-orthogonality in Rickart rings. .

David Jacobson and Kenneth S. Williams, On the solution of linear G.C.D.

equations

Michael Joseph Kallaher, On rank 3 projective planes ... . .

Donald Paul Minassian, On solvable $O^{*}$-groups ...........

Nils Øvrelid, Generators of the maximal ideals of $A(\bar{D})$

Mohan S. Putcha and Julian Weissglass, A semilattice decomposition into

semigroups having at most one idempotent ............

Robert Raphael, Rings of quotients and $\pi$-regularity ....

J. A. Siddiqi, Infinite matrices summing every almost periodic sequence. .

Raymond Earl Smithson, Uniform convergence for multifunctions ...

Thomas Paul Whaley, Mulitplicity type and congruence relations in

universal algebras...

Roger Allen Wiegand, Globalization theorems for locally finitely generated modules... 